\section{Bei Malignomen: Individualisierte Antikoagulation der Thromboembolie}

\begin{abstract}
Durch Fortschritte in der Therapie bösartiger Tumoren hat sich die Lebenserwartung von Krebspatienten inzwischen verlängert. Jetzt sollte auch die Behandlung tumorassoziierter Komplikationen optimiert werden.
\end{abstract}

\begin{abstract}
$\mathrm{n}$ ein internationales Register werden konsekutiv alle Patienten mit gesicherter tiefer Beinvenenthrombose und/oder Lungenembolie aufgenommen. Darunter befinden sich 3.947 Patienten mit den vier häufigsten Karzinomen (938 Mamma-, 629 Prostata-, 1.189 Kolorektalund 1.191 Bronchialkarzinome). Etwa $50 \%$ der Patienten hatten eine Lungenembolie, bei 11-43\% war das Malignom erst innerhalb der letzten drei Monate diagnostiziert worden, bei $55 \%$ waren Metastasen bereits nachweisbar.

$\mathrm{Zu}$ Beginn der Antikoagulation über im Mittel 139 Tage standen 50\% unter einer Chemo- und $15 \%$ unter einer Radiotherapie. Die Antikoagulation erfolg-
\end{abstract}

te nach den Präferenzen in den einzelnen Kliniken, am häufigsten mit niedermolekularem Heparin und Vitamin-AAntagonisten, teils auch mit Rivaroxaban.

Das Verhältnis von erwünschten und unerwünschten Wirkungen der Antikoagulation war bei den einzelnen Malignomen sehr unterschiedlich (Tab. 1). So traten Thromboembolierezidive beim Lungenkarzinom mit fünffacher, schwere Blutungen bei den drei anderen Karzinomen mit etwa dreifacher Häufigkeit auf als beim Mammakarzinom. Die Zahl neuer Thromboembolien und schwerer Blutungen waren bei Mammaund kolorektalem Karzinomen gleich. Beim Prostatakarzinom überwogen die

Tab. 1: Thromboembolierezidive und schwere Blutungen unter Antikoagulation bei Krebspatienten (pro 100 Patientenjahre)

\begin{tabular}{|l|l|l|l|l|}
\hline & $\begin{array}{l}\text { Mamma- } \\
\text { karzinom }\end{array}$ & $\begin{array}{l}\text { Kolorektal- } \\
\text { karzinom }\end{array}$ & $\begin{array}{l}\text { Prostata- } \\
\text { karzinom }\end{array}$ & $\begin{array}{l}\text { Lungen- } \\
\text { karzinom }\end{array}$ \\
\hline neue Thromboembolien & 5,6 & 10 & 6,9 & 27 \\
\hline schwere Blutungen & 4,1 & 12 & 13 & 11 \\
\hline
\end{tabular}

\section{Späte Stentthrombosen bei Scaffolds}

\section{Wie gut sind absorbierbare Stentgerüste (Scaffolds) im Vergleich zu} medikamentenbeschichteten Stents (DES)? Die aktuellen Daten geben Aufschluss über das Risiko für Stentthrombosen.

A bsorbierbare Stents können möglicherweise Re-Interventionen/-Operationen technisch erleichtern (z.B. bei Bifurkationläsionen), nicht invasive Kontrolluntersuchungen (z.B. Computertomografie der Koronarstents) ermöglichen und gegebenenfalls auch vaskulär adaptative Prozesse günstig beeinflussen (Vasomotion, Remodeling).
Für den klinisch derzeit am besten erprobten absorbierbaren Stent (bioresorbable vascular scaffold, BVS) ABSORB liegen derzeit Daten aus sechs ter Einschluss von 3.708 Patienten vor. In einer aktuellen Publikation wurden die Sicherheit und Effektivität des BVS in fünf randomisierten Studien (1.730 randomisierten klinischen Studien un- schweren Blutungen, beim Lungenkarzinom die Thromboembolierezidive.

Mahé l et al. The Clinical Course of Venous Thromboembolism May Differ According to Cancer Site. Amer J Med. 2017;130:337-47

\section{Kommentar}

Akute venöse Thromboembolien bei Patienten mit bösartigen Erkrankungen sind häufig. Die Leitlinien empfehlen eine Antikoagulation für mindestens drei Monate, unabhängig von Lokalisation und Art des Tumors, seiner Ausbreitung und der spezifischen Tumortherapie. Das Thromboembolierisiko wird aber von diesen Eigenschaften beeinflusst, doch fehlen entsprechende Therapiestudien.

Die vorliegende, umfangreiche Beobachtungsstudie mit konventioneller, aber nicht standardisierter Antikoagulation zeigt deutlich, dass das Thromboembolie- und Blutungsrisiko bei den vier häufigsten Karzinomen unterschiedlich ist. Diese Ergebnisse könnten dazu beitragen, die Therapie nach Tumorlokalisation zu individualisieren und zu verbessern. Ansatzpunkt sind die hohen Raten von Thromboembolien bei Lungenkarzinomen und von Blutungen bei Prostatakarzinomen.

Prof. Dr. med. Heinrich
Holzgreve
Kardiologische Praxis
München

Patienten) mit einer Nachbeobachtungsdauer von mindestens 24 Monaten mit der von aktuellen Everolimus eluierenden DES verglichen. Die Analyse bezog sich zum einen auf das Risiko von Stentthrombosen, zum anderen wurde hinsichtlich der Effektivität das Versagen am Zielgefäß (target lesion failure, TLF) untersucht.

Patienten mit dem BVS hatten eine höhere Wahrscheinlichkeit für Stentthrombosen (OR 2,93; $p=0,01$ ), einschließlich sehr später Stentthrombosen (13 Patienten). Die Mehrzahl der sehr späten BVS-Thrombosen trat unter einer plättchenhemmenden Monotherapie 